\title{
Credentialing medical education: how much education do you need to be a good
}

teacher?

Faye Gishen, Deborah Gill, Jane Dacre

Dr Faye Gishen, BSc MBBS FRCP SFHEA FAcadMedEd is a consultant in palliative medicine and honorary senior lecturer at UCL Medical School f.gishen@ucl.ac.uk

Professor Deborah Gill MBBS FRCGP EdD PFHEA is a general practitioner, Professor of medical education and Director of UCL Medical School

Professor Dame Jane Dacre DBE MD FRCP is a consultant rheumatologist, Professor of medical education and immediate past President of the Royal College of Physicians, UK

All authors are credentialed medical educators. 
At a recent UK meeting about medical education, a keynote speaker said in a talk entitled 'The Credentialed Medical Educator';

"If you want to be a good medical educator you need to be able to teach" (1)

Whilst medical education will always rely heavily on enthusiastic clinicians as the bedrock of delivering clinical teaching, the age of the 'on-the-job' clinician designing complex medical curricula is surely over. Traditionally, medical education at both undergraduate and postgraduate level, has drawn on keen and willing clinicians to design programmes and pedagogical approaches as well as to deliver teaching. In contrast, our clinician scientist colleagues, whilst relying on fellow clinicians to recruit patients to clinical trials, would not expect them to determine methodology or analyse data. In order to champion an equivalent ethos, the clinical academics amongst us need to be promoting a specialist approach to medical education. Those in positions of building, curating or leading medical curricula should be selected for their experience and expertise supported by further study in education.

This is not based on establishing a 'protectionist' approach to medical education, but rather one grounded in knowledge. Primary and secondary education is designed and driven by dedicated educationalists who require pedagogical knowledge in teaching theory and methods, such as the Postgraduate Certificate in Education (PGCE). As a profession, we can no longer justify our approach to preparing generations of medical students for professional practice without embedding this in deeper understanding of educational theory and underpinning what and how we are teaching with robust evidence. Medical educational research is as important as clinical research and the stakes are just as high, when we consider that we are preparing thousands of future doctors for the responsibility and professional duties of caring for patients. The impact on citizens and society of poor quality, under- 
researched education of its doctors is potentially huge. We would argue that we should apply equal rigour to the educational arena to that which we apply to clinical practice, and this should be an aspiration for the profession.

The complexities of clinical medicine are mirrored in the sphere of medical education, as content, methodologies and technology become more sophisticated. Preparing our future doctors and subsequently training them in the postgraduate space, requires specialist and dedicated medical educators having a significant educational background themselves. Granted, collaboration with clinical teachers from a range of disciplinary backgrounds is vital, in order to produce operationalizable curricula, but these should be fundamentally designed and dynamically curated by those with dedicated educational skills and knowledge. A recent article (2) critically argues for this;

"In our daily lives as clinicians, we aim to create a culture of continuous quality improvement. We should strive to create the same culture in our educational lives"

A 2018 Royal College of Physician report (3) describes 'Doctor as learner and teacher' as one of seven core characteristics constituting medical professionalism. Historically however, teaching within medicine has been viewed by many as having lower status than both clinical work and research. Dedicated time for teaching and support of learning can be sacrificed in the climate of busy clinical roles. This article is not intended to discredit the thousands of highly valued and effective professionals conscientiously teaching medical students and junior medical colleagues throughout the UK and the world. However, the increasing demands to preserve and protect clinical commitments can result in fewer opportunities for 
clinicians to contribute to broader activities such as educational planning and review, assessments and instructional material design.

Viewing one's contribution to medical education through a different lens may be helpful; teaching medical students and junior doctors may be professionally nourishing and symbiotic. Being involved in teaching and training could enhance job satisfaction, clinician wellbeing and the morale of our workforce. Encouragingly, in the era of the 'portfolio career', many doctors report an interest in teaching and pursuing a credential in medical education (4). Many feel that having a credential could contribute to enhancing role recognition and to raising standards of medical education. Other contributions could include career development, networking and enhanced promotion prospects. Some however, question whether a credential would actually make them better medical educators. We would argue that pursuing further studies in education can contribute evidence of scholarship in this domain.

Current opportunities for credentialing and learning amongst doctors include local faculty development courses (such as Training to Teach modules) and undertaking diplomas or certificates in medical education. Such opportunities could be ideal for clinicians with a particular interest in teaching. Those with wider medical education aspirations could consider undertaking a masters or doctoral level degree (such as a $\mathrm{PhD}$ or professional doctorate) (5). There are also opportunities to gain credentials in teaching through membership of national or international education excellence organisations (such as American, Australian or European Associations for Higher Education). There are opportunities to showcase educational scholarship through publication of educational research in medical education (or broader) journals and conferences, developing education policy or contributing to expert 
advisory groups. By approaching medical education with an increasingly scholarly and formalised ethos, we will ensure that it is regarded as a bone fide specialty in its own right.

(words 850)

References

(1) Erik Driessen, Maastricht University, at 'Developing a Credential in Medical Education' hosted by Wales Deanery, March 2016

(2) Schwartzstein, R, Roberts D. Saying goodbye to lectures in medical school- paradigm shift or passing fad? N Engl J Med 2017; 377:605-607

(3) https://www.rcplondon.ac.uk/news/medical-professionalism-essential-needs-moresupport-says-rcp

(4) Pathiraja F, Wilson M-C. The rise and rise of the portfolio career. BMJ 2011; 342

(5) Gill D, Griffin A, Woolf K, Cave K. Twelve tips for studying medical education at doctoral level, Medical Teacher, 2009: 31:7, 601-604 